\title{
Usefulness of realignement and "phase analysis" in the assessment of the synchronism of regional left ventricular contraction and relaxation
}

Melchior, J. P. (Geneva); Doriot, P. A.; Chatelain, P.

Cardiology Center, University Hospital, CH-1211 Geneva 4

\author{
Introduction
}

A great variety of models have been proposed for the quantification of the left ventricular regional contraction from angiographic sequences. Most of them involve an enddiastolic/endsystolic image pair only, but could be extended without additional conceptual difficulties to all silhouettes of a selected cardiac cycle. Comparisons of the different existing models have been performed by various groups on large samples of control subjects and patients, but consensus on the best approach is still not obtained.

The aim of the present study was not to demonstrate the superiority of our model (described in the following). Nevertheless, we believe that it does not present certain (in our judgement) unsatisfying aspects of some chordal, radial or "center-line" derived models.

Our main goal was rather to investigate following point which, in our opinion, should be clarified in priority and conclusively:

Should the silhouettes be repositioned relatively to the enddiastolic silhouette (for instance) previously to any quantification, in order to eliminate as well as possible translation and rotation components of the heart motion during the cardiac cycle, relatively to the $x$-ray imaging system ?

The results obtained in our patient studies may constitute a valuable answer to this key question.

\section{A Criterion for the Usefulness of Realignement}

From a physical point of view, it is obvious that translation and rotation of the left ventricular cavity in the image during the cardiac cycle should be compensated, as one would eliminate such motion components to study the oscillatory deformation of a kicked, flying rugby ball. This is equivalent to say that the contraction pattern of the left ventricle (LV) should be considered as shape modification of the silhouette rather than as apparent "wall motion". Putting the weight onto the shape leads to design a model in which the shape specificity of the LV silhouette plays a dominant role. This in turns leads to let a caracteristic point of the valvular region and the apex govern the realignement of each silhouette, as would the 2 poles of

\begin{abstract}
the rugby ball define the orientation of the adequate coordinates system.

However, even evidences must be supported by experimental results in agreement with a well accepted postulate. Such a postulate fortunately exists: it states that left ventricles of control subjects contract and relax essentially in a synchrone fashion (when refered for instance to the mid-aortic point). The "phase analysis" technique is an ideal tool for the investigation of contraction/relaxation synchronism since its basic criterion is precisely the degree of synchronism of the LV contraction/relaxation. Superiority of realignement compared to non-realignement can thus be assessed by showing that it yields higher synchronism of the contraction/relaxation in control subjects.
\end{abstract}

our contraction/relaxation model includes therefore preliminar realignement of the silhouettes (right anterior oblique 30 projection) by use of midaortic point and silhouette long axis, and "phase analysis" of a complete cardiac cycle.

\section{The Method}

Beginning with the enddiastolic silhouette, the contours of all silhouettes of the selected cardiac cycle are drawn on mask subtracted images $(25 \mathrm{i} / \mathrm{s})$ and stored in a VAX-11/750 computer (Enddiastole is defined by the $R$ peak of the ECG). These native contours are then realigned by superimposition of the mid-aortic points and of the "long axis" of the silhouettes. The "long axis" of each silhouette is defined by the mid-aortic point and an apical point determined in following way (Fig. 1): First the usual long-axis of the silhouette through the midaortic point and the farthest contour point is determined; then a line perpendicular to this first axis is drawn at $19 / 20$ ths of its length (measures from the mid-aortic point). This auxillary line defines a small apical area, whose center of gravity is the second point of our new "long-axis" (This reduces the variability of apex determination).

The first (enddiastolic) contour is then divided by $2 \times 64$ equidistant points: 64 hefore and 64 after the apex (anterior, respectively inferior region of the iv). Linking these 128 points to the corresponding points of all following contours creates 128 shor- 


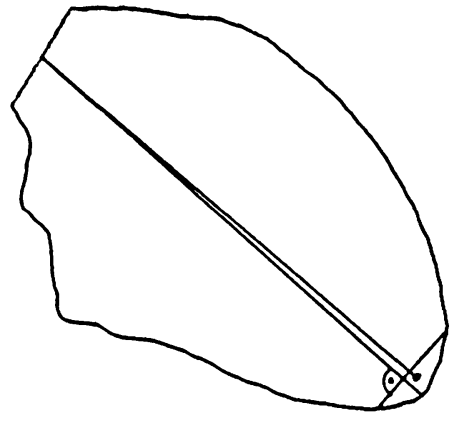

Figure 1: Principle of silhouette realignement.
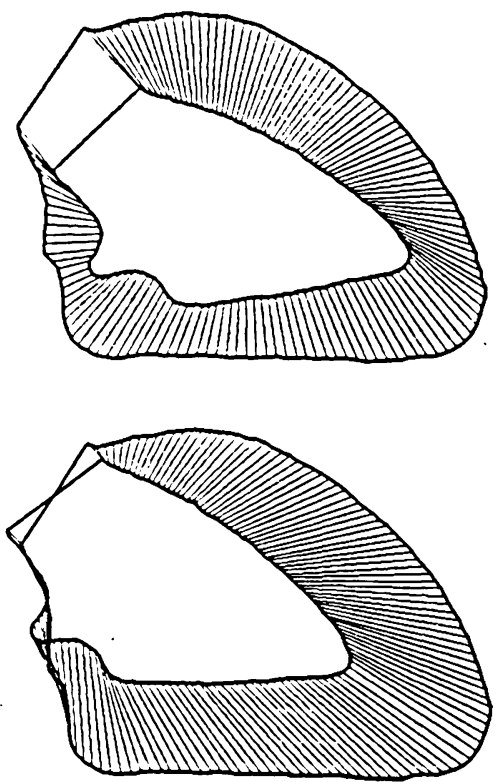

Figure 2: Enddiastolic/endsystolic silhouette pair, before and after realignement.

tening segments (Fig. 2). "Phase analysis" is performed on the selected cardiac cycle as follows: For each segment a contraction-relaxation timecurve is obtained by a 2-harmonics Fourier fit of its measured lengths in the course of cardiac cycle (Cardiac cycle duration is normalized to 360 ). The phase (Pha) of the first harmonic is the first parameter obtained. Two other parameters, the "time of peak contraction" (TPC) and the "time of peak relaxation" (TPR), are defined as the times of the maximum respectively of the minimum value of the first derivative of the fitted curve. Thus, 3 parameters expressed in degrees are assigned to each segment, each one reflecting an aspect of the timeevolution of the segment during the cardiac cycle.
The segments are color coded according to the "value of the considered paramoter and displayed at their respective location in the enddiastollc/endsystollc sllhouettes palr. This ylelds 3 parametric images. The histogram of each parameter $1 \mathrm{~s}$ also computed, with mean and standard deviation (SD).

\section{Study on Control subjects}

We have first studied 13 control subjects (C) without and with contour realignement. In our experience the means of the 3 histograms are not useful. But the 3 SD, which reflect the degree of synchronism of the contraction or relaxation, show clearly $(p<0.02)$ ) that the postulated synchronism of left ventricular contraction and relaxation in control subjects is better reflected if realignement is performed:

\begin{tabular}{llrr}
$C(13)$ & $\overline{S D}$ of Pha & $\overline{\text { SD }}$ of TPC & \multicolumn{1}{c}{$\overline{\text { SD }}$ of TPR } \\
\hline Not realigned & $9.2 \pm 4.0$ & $15.1 \pm 10.8$ & $14.9 \pm 9.9$ \\
Realigned & $5.8 \pm 2.3$ & $6.5 \pm 2.0$ & $6.5 \pm 2.1$
\end{tabular}
Realigned $5.8 \pm 2.3$
SD: mean standard deviations

The standard deviations of the $\overline{S D}$ are also lower after realignement, which is a further. finding -quite in agreement with the postulate of high synchronism in control subjects.

\section{Study on Patients}

Sixteen patients with a proven myocardial infarction (MI) were studied next. As can be seen in the table below, all 3 SD appear to be sensitive indicators of pathological states when compared to the SD of normal subjects:

\begin{tabular}{lccc} 
MI (16) & $\overline{\text { SD }}$ of Pha & $\overline{\text { SD }}$ of TPC & SD of TPR \\
\hline Not realigned & $23.3 \pm 16.2$ & $26.8 \pm 16.1$ & $33.4 \pm 14.4$ \\
Realigned & $13.9 \pm 7.9$ & $13.2 \pm 7.5$ & $23.8 \pm 12.9$
\end{tabular}
SD: mean standard deviations

The SD in this group of MI patients are also reduced by realignement, but, unlike to the case of - control subjects, this is not relevant. However, a further interesting result consists in the decrease of the standard deviations of the $\overline{S D}$ in the sense that it reflects less overlap of the individual SD values between the MI and C groups. Realignement allows thus for a better differentiation of MI patients from normal subjects, whereby the parameter TPR appears to be more sensitive than the 2 others.

Regional amplitude of contraction was also studied in these 16 patients. After realignement hypokinesia was detected, in the 16 infarcted territories corresponding to the occluded coronary artery. without realignement hypokinesia was not detected in one patient and localized in a. wrong territory (unrelated with the occluded vessel and the ECG $Q$ waves) in 2 patients.

\section{Conclusion}

Realignement of contours provides in control subjects a higher homogeneity of each 3 parameters in agreement with the basic postulate that left ventricles of normal subjects contract and relax in a synchrone fashion. Realignement results also in more sensitivity in detection and localization of asynchronism, and rechuces erroneous localization of hypokinesia. The parameter TPR appears to be more sensitive than the 2 others for the detection of ischemic states. 